Ramírez, A., Arenas, L. (2016). Tipificación técnica y socioeconómica de trapiches paneleros en el municipio de Ocamonte, Santander: Una aplicación de análisis multivariado. Revista Lebret, 8. Bucaramanga, Colombia: Universidad Santo Tomás, pp. 243 - 261. ISSN: 2145-5996

\title{
Tipificación técnica y socioeconómica de trapiches paneleros en el municipio de Ocamonte, Santander: una aplicación de análisis multivariado*
}

\section{Technical and socio economic classification of panela farms in Ocamonte municipality of Santander Department: an application of multivariate analysis}

\author{
Álvaro Ramírez Suárez \\ Leidy Ximena Arenas Mejía
}

\begin{abstract}
Resumen
Los trapiches paneleros se caracterizan por una alta heterogeneidad en las características socioculturales de los productores, estructura productiva, dotación de recursos productivos y uso de tecnología. En este estudio se aplicaron y validaron técnicas de análisis estadístico multivariado (análisis de correspondencia múltiple con escalamiento óptimo, análisis de conglomerados y análisis discriminante) como metodología para conformar grupos homogéneos de trapiches a partir de una muestra de 43 trapiches del municipio de Ocamonte, Santander. Se encontraron cuatro tipos de trapiches los cuales son representativos de tres sistemas de producción de panela bien diferenciados. El sistema marginal que, comprende el 65\% de los trapiches (trapiches Tipo I). Un sistema tradicional mejorado (7\%) (trapiches Tipo III). Finalmente, un sistema avanzado (28\%) (trapiches tipos II y IV). Para mejorar su eficiencia productiva estos tipos de trapiches deben intervenirse mediante líneas de política diferenciadas.
\end{abstract}

Palabras clave

Panela, trapiche, tipificación, análisis multivariado.

Código de clasificación JEL: L66, Q12

\begin{abstract}
Brown sugar mills are characterized by their high heterogeneity regarding producers' sociodemographic features, mills productive structure, resource endowment, technology use and economic performance. In order to test and validate a methodology to determine homogeneous groups of brown sugar mills, a random sample survey of 43 mills was researched at Ocamonte, Santander. To accomplish research's main objectives a sequential multivariate statistic approach was applied (namely, multiple correspondence analysis with optimum scaling, cluster analysis and discriminant analysis). There were found four well differentiated types of mills representing three brown sugar production systems namely: a marginal (Type I) system encompassing $65 \%$ of mills in the sample. Another traditional improved (Type III) system involving 7\% of mills. Finally, an advanced (Type II and IV) system embracing $28 \%$ of mills. Improving the productive efficiency of these mill types requires different sorts of policy interventions.
\end{abstract}

\section{Keywords}

Brown sugar, brown sugar mill, typology, multivariate statistic analysis.

* Artículo derivado del proyecto de investigación: "Estudio piloto para la tipificación técnica y socioeconómica de trapiches paneleros en el municipio de Ocamonte, Santander, con la participación de la Universidad Santo Tomás Seccional Bucaramanga y Fedepanela.

1 Doctor of Philosophy Agricultural Economics. Docente facultades de Administración de Empresas Agropecuarias y Economía en la Universidad Santo Tomás Seccional Bucaramanga. Correo electrónico: alvaro.ramirez@ ustabuca.edu.co

2 Economista, investigadora de la Federación Nacional de Cultivadores de Panela FEDEPANELA. Correo electrónico: ximenarenas92@gmail.com 


\section{Introducción}

En la agricultura, las técnicas de agrupación multivariadas han sido útiles en estudios socioeconómicos a fin de establecer:

1. La respuesta diferencial de las unidades productivas (agricultores, sistemas de fincas, sistemas de cultivo) con respecto a una política económica no diferenciada, por ejemplo: generación y transferencia de tecnologías aumentadoras de la productividad o reducidoras de costos (Winkler, 1977), (Calvo \& Icaza, 1988), (Capillon, 1986), (Suárez \& Escobar, 1990), (Carvajal, Pinzon, \& Restrepo, 1992), (Dussan, 1996), (Isaacs y otros, 1998), (Aguiar, 2001), (Madry y otros, 2013).

2. La diferenciación de política necesaria para obtener una respuesta dada, como: incentivos a la tasa de interés, incentivos de precios de insumos y productos, estímulos a la redistribución de tierras, incentivos a programas de apoyo al ingreso de pequeños productores (Pretzer \& Finley, 1974), (Cohan \& Alonso, 1977), (Kaminski, 1982), (Manyong, Degand, D’ Haese, \& Dutilleul, 1988), (Escobar \& Berdegué, 1990), (Mantilla, Arguello, \& Méndez, 2000), (Rios, 1999), (Rios, y otros, 2002), (Smith, Moreira, \& Latrille, 2002).

El alcance de las técnicas multivariadas depende del avance en el conocimiento que se tenga, producto de la investigación sistemática, de la realidad técnica y socioeconómica de las unidades productivas a lo largo de las cadenas de valor en la agricultura (agricultores, hogares, fincas o predios, unidades de transformación, distribución y consumidores). En Colombia las aplicaciones sobre tipificación de unidades productivas son muy escasas en la última década. Sin embargo, estas tienen relevancia estratégica bajo el principio aceptado de que, la definición de unidades productivas tipo, es esencial para una correcta y eficiente definición de políticas socioeconómicas (Kaminski, 1986). Por ejemplo, el sector panelero colombiano adolece de un sistema de información básico, en tiempo real, sobre la estructura de las fincas y trapiches paneleros y el desempeño técnico y socioeconómico de los agricultores productores de caña de azúcar y de panela. Esto ha venido limitando la capacidad de los agentes económicos ${ }^{3}$ para tomar decisiones informadas que tengan altas probabilidades de hacer impacto sobre el bienestar de los agricultores, sus familias, y las comunidades y regiones paneleras.

Una de las causas de esta problemática se atribuye a la falta de metodologías efectivas en costos, uniformes y comparables, probadas y validadas para generar datos básicos sobre la realidad del campo colombiano (DANE, 2009), (Acosta \& Pérez, 2011), (Fedesarrollo-Iquartil-SAC, 2013). Estos datos deben caracterizar la alta heterogeneidad existente en las condiciones agroecológicas de las unidades

3 Agricultores, técnicos, administradores de servicios de apoyo (investigación, transferencia de tecnología, financiamiento, crédito, mercados, bienes públicos) y formuladores de política económica. 
productivas y socioeconómicas de los agricultores, la dotación de recursos, servicios productivos, las tecnologías de producción y los bienes públicos existentes en las zonas rurales. Esta información es esencial para: a) entender las limitantes estructurales, de funcionamiento y desempeño de la producción, ingresos y bienestar de los productores; b) entender su dinámica en el tiempo; c) finalmente, para diseñar programas y estrategias de política económica y social relevantes para superarlas. Así lo recomiendan recientemente: el Banco Mundial (World Bank, 2009), la Organización de las Naciones Unidas (PNUD, 2011) y la Misión Rural para la Transformación del Campo Colombiano (DNP-MTC, 2015).

En Ocamonte hay un exceso de capacidad instalada para la elaboración de la panela. Las fincas productoras de caña panelera y con trapiche en esta zona presentan un déficit crónico de caña para utilizar la capacidad instalada de los trapiches. La superficie en caña es reducida con relación al número de trapiches existentes. Según la encuesta de Evaluación Agropecuaria 2013, el municipio reportó 1213 hectáreas cosechadas en ese año. En contraste, el número total de trapiches censados era de 54 unidades. En consecuencia, el promedio de área en caña se estima en 23 hectáreas por trapiche para una producción promedia anual de 70 ton-año de panela por trapiche. Dos terceras partes de los trapiches estudiados producen este tonelaje de panela al año, pero su capacidad de molienda es mayor a 10 ton - hora. Se configura así, un exceso de capacidad instalada de molienda que implica un alto lucro cesante, altos costos unitarios de producción de panela, menores ingresos y ganancias para los productores y mayores pérdidas de bienestar para las comunidades paneleras. Por tanto, se requiere caracterizar estos trapiches a fin de encontrar alternativas de política adecuadas para mejorar su eficiencia productiva actual.

En este estudio se discuten los resultados de la aplicación de una metodología para la caracterización de las condiciones de producción de panela en el municipio de Ocamonte, Santander, con el fin de conformar grupos homogéneos de trapiches bien diferenciados entre sí, usando técnicas de análisis multivariado. No se conocen estudios publicados de tipificación de trapiches paneleros en Colombia. El análisis asume que las respuestas de los agricultores a los incentivos y desestímulos de la política socioeconómica y los mercados son diferenciados, según sean las características socioculturales y económicas del productor y técnico financieras de la unidad productiva (Kaminski, 1986). Así, la tipificación estadística retoma su relevancia táctica como instrumento de diferenciación de unidades productivas y estratégica en la definición de líneas de acción de política social, económica y sectorial para la agricultura colombiana ${ }^{4}$.

4 Durante el período 1970-1990, el Instituto Interamericano de Cooperación para la Agricultura de la OEA-IICA y el actual Centro Internacional de Desarrollo Rural RIMISP, promovieron el uso de las técnicas de análisis multivariado en América Latina y el Caribe para apoyar la definición de políticas (Arenas, 2013). 


\section{Métodos y procedimientos}

La tipificación como técnica de aglomeración usando análisis estadísticos multivariados, forma parte del método científico y se aplica para probar la hipótesis de que, la población en conjunto, no es homogénea para los fines de diferenciación de las intervenciones de política. Por tanto, es un medio para identificar estructuras productivas y socioeconómicas diferenciadas que requieren ser intervenidas. Esta conclusión trivial no siempre se deriva de las tipificaciones convencionales, las cuales usan métodos estadísticos univariados o bivariados, y pueden resultar en unidades productivas "tipo" poco o mal diferenciadas respecto a los propósitos del análisis (Kaminski, 1982).

Para adelantar la presente investigación se usó información censal de corte transversal compuesta por 43 trapiches paneleros, equivalentes al $80 \%$ de los trapiches localizados en el municipio de Ocamonte, departamento de Santander ${ }^{5}$. Los datos se levantaron mediante la aplicación de una encuesta estructurada al productor en entrevista personal y observación directa del trapiche. El contenido del formato de encuesta reflejaba el supuesto de que, los productores de panela en esta zona son relativamente heterogéneos en términos: socioeconómicos y sociodemográficos, el acceso a servicios productivos, la dotación de recursos y el sistema de producción.

El estudio se enfocó en el análisis de estas cuatro grandes dimensiones, las cuales resaltan características particulares del agricultor y la producción panelera. Más específicamente, en relación con atributos como: la edad, experiencia en el cultivo de caña y en el proceso de elaboración de panela, nivel de educación, composición familiar, tamaño y tenencia del cultivo de caña y los trapiches, intensidad de uso anual del trapiche y su capacidad instalada. Además se incluyeron variables indicadoras de la dotación y uso de recursos y servicios productivos (tierra y su tenencia, instalaciones, agua, electricidad, saneamiento básico, mano de obra, fuerza animal, capital, tecnología, crédito, mercados, y desempeño técnico y económico de los trapiches (Arenas, 2013).

El modelo empírico de tipificación se construyó en cuatro fases. En la primera, se hizo un análisis estadístico descriptivo y exploratorio de la calidad de los datos y de las variables cuantitativas y cualitativas (en escala nominal y ordinal) que, en teoría y con base en el conocimiento de los investigadores, tendrían poder clasificatorio. Esto con el fin de: detectar errores en los datos, identificar valores extremos, crear nuevas variables ${ }^{6}$, identificar el grado de interdependencia de las variables y asegurar las condiciones de linealidad, normalidad y homocedasticidad requeridas por las técnicas de análisis multivariados. Para adelantar la selección de variables cuantitativas

5 La población de trapiches en Ocamonte era de 54 unidades en el 2013. La ausencia de los productores durante la visita de campo fue la principal razón para no incluir todas las unidades productivas en el estudio.

6 Algunas de estas variables se transformaron usando criterios de "equivalencias" para obtener por ejemplo: el número de horas-hombre-año equivalente, horas-animal-año equivalente y horas-molienda-año equivalente, un indicador de ocupación del trapiche entre otras (Arenas, 2013). 
clasificatorias, estas se estandarizaron por su valor de $\mathrm{Z}$ y luego se cotejaron en pares mediante análisis de correlación lineal simple (correlación de Pearson).

En la segunda fase, se adelantó un análisis de correspondencias múltiple ACM con escalamiento óptimo a fin de representar en un espacio multidimensional reduciendo la relación existente entre las variables de tipo nominal y ordinal (cualitativas) con poder clasificatorio, seleccionadas en la fase anterior (Hair, Anderson, Tatham \& Black, 1998). Al efecto, las variables cuantitativas identificadas como clasificatorias se integraron al análisis mediante su recodificación previa en variables nominales ordinales. De este modo, no solo se redujo la dimensionalidad de las variables seleccionadas, sino también: a) se examinó la información aportada por cada observación (trapiche) a cada dimensión; b) se compararon las unidades productivas (trapiches) según las variables clasificatorias y sus relaciones; b) se resumió el conjunto de variables seleccionadas en un conjunto pequeño de variables cuantitativas; c) se definieron y examinaron los ejes $(\mathrm{X}, \mathrm{Y})$ de dispersión o factores, seleccionando las combinaciones de variables mejor correlacionadas; y d) se proyectaron gráficamente los trapiches y la variables de clasificación en el plano bidimensional, facilitando así su interpretación.

Una vez reducida la dimensionalidad, en la tercera fase se hizo un análisis de conglomerados usando la técnica de agrupamiento jerárquico para clasificar los trapiches, empleando los factores o dimensiones seleccionadas en la fase anterior (Johnson \& Williamson, 1998). Este método se seleccionó dado que no se tenía suficiente conocimiento a priori sobre el número de grupos de trapiches por conformar. En este caso, se generaron dendogramas o diagramas de árbol mediante los cuales se conformaron los grupos de trapiches (clústeres) ${ }^{7}$. El método permitió agrupar las observaciones en conjuntos bien diferenciados entre sí pero muy similares dentro de cada grupo. Mientras que, las técnicas de análisis de conglomerados realizan y proponen la clasificación, el número de grupos de trapiches "tipo" final resultó de la interpretación que los investigadores hicieron de los conglomerados resultantes, basados en el conocimiento real de las unidades en agrupación y su validación en el campo.

En la cuarta fase se adelantó un análisis discriminante con el propósito de comprobar la calidad de los agrupamientos resultantes y validar como correcta la asignación de los trapiches dentro de los grupos conformados (Chatfield \& Collins, 1992). Realizada la validación estadística de los grupos de trapiches resultantes, se hizo un análisis descriptivo de los respectivos sistemas de producción dentro de cada tipo de trapiches. Con este fin, dentro de cada grupo, se realizó una caracterización general de cada uno de los procesos de producción de panela con relación a la tecnología empleada en los trapiches (prácticas de manejo técnico de las operaciones y sus procesos). Así mismo, se compararon las principales

7 El dendograma es una representación gráfica que muestra cómo los grupos de niveles inferiores se engloban jerárquicamente a otros grupos en niveles superiores. 
características sociodemográficas y culturales de los productores, así como, la dotación de recursos, servicios y desempeño técnico económico de los distintos tipos de trapiches encontrados.

\section{Análisis de resultados}

\section{Selección de variables clasificatorias}

Como se ilustra en la tabla 1, las variables seleccionadas como clasificatorias correspondieron a las dimensiones: sociodemográfica (6 variables) y estructura productiva (20 variables sobre dotación de recursos, acceso a servicios productivos y tecnología). Para la aplicación de las técnicas de análisis multivariado se construyó una matriz de datos constituida por 26 variables seleccionadas y 43 observaciones (trapiches).

Tabla 1. Principales variables seleccionadas como clasificatorias, según dimensiones

\begin{tabular}{|c|c|c|c|c|c|}
\hline Variables & $\begin{array}{c}\text { Tipo de } \\
\text { variables }\end{array}$ & $\begin{array}{l}\text { No. } \\
\text { categorías }\end{array}$ & Variables & $\begin{array}{c}\text { Tipo de } \\
\text { variables }\end{array}$ & $\begin{array}{l}\text { No. } \\
\text { categorías }\end{array}$ \\
\hline \multicolumn{3}{|c|}{ Dimensión sociodemográfica } & \multicolumn{3}{|c|}{ Dimensión socioeconómica y productiva } \\
\hline \multirow{2}{*}{$\begin{array}{l}\text { Tiempo dedicación } \\
\text { productor al trapiche }\end{array}$} & \multirow{2}{*}{ Nominal } & \multirow{2}{*}{2} & $\begin{array}{l}\text { M.O familiar permanente no } \\
\text { remunerada }\end{array}$ & Nominal & 2 \\
\hline & & & $\begin{array}{l}\text { Mano de obra permanente } \\
\text { contratada remunerada }\end{array}$ & Nominal & 2 \\
\hline \multirow{2}{*}{ Edad } & \multirow{2}{*}{ Ordinal } & \multirow{2}{*}{5} & Fuente oferta cañas & Nominal & 2 \\
\hline & & & Área sembrada en caña panelera & Ordinal & 5 \\
\hline Sexo & $\begin{array}{c}\text { Cualitativa } \\
\text { nominal }\end{array}$ & 2 & $\begin{array}{l}\text { Mano obra ocasional remunerada } \\
\text { equivalentes hombre año total }\end{array}$ & Intervalo & 1 \\
\hline \multirow{3}{*}{$\begin{array}{l}\text { Años de tenencia } \\
\text { trapiche }\end{array}$} & \multirow{3}{*}{ Ordinal } & \multirow{3}{*}{4} & $\begin{array}{l}\text { Fuerza animal equivalentes } \\
\text { animal año total }\end{array}$ & Intervalo & 1 \\
\hline & & & $\begin{array}{c}\text { Capacidad hornilla kg panela/ } \\
\text { hora }\end{array}$ & Ordinal & 5 \\
\hline & & & Toneladas caña propia & Intervalo & 1 \\
\hline \multirow{2}{*}{$\begin{array}{l}\text { Años experiencia } \\
\text { productor }\end{array}$} & \multirow{2}{*}{ Ordinal } & \multirow{2}{*}{6} & Toneladas caña fincas vecinas & Intervalo & 1 \\
\hline & & & Toneladas caña totales & Intervalo & 1 \\
\hline \multirow[t]{5}{*}{ Distancia en $\mathrm{km}$} & Intervalo & 1 & Semanas molienda totales año & Intervalo & 1 \\
\hline & & & Toneladas de panela año & Intervalo & 1 \\
\hline & & & Separación bagazo evaporación & Nominal & 2 \\
\hline & & & Área construida $\mathrm{m}^{2}$ & Intervalo & 1 \\
\hline & & & Valor activos fijos trapiche $\$$ & Intervalo & 1 \\
\hline
\end{tabular}

Fuente: Arenas, 2013 


\section{Análisis de correspondencias múltiples ACM}

El análisis de correspondencias múltiples es una técnica para examinar estadísticamente el grado de dependencia o independencia entre las categorías de variables cualitativas nominales y ordinales (Clausen , 1998). El modelo representa gráficamente la estructura de las relaciones entre más de dos variables mediante mapas de posicionamiento. Al igual que en el análisis de correspondencias simples, las relaciones entre las categorías de las variables se analizan a través de los mapas perceptuales, donde cada categoría es ubicada en un punto (Calvo \& Icaza, 1988). La distancia $\left(\mathrm{X}^{2}\right.$, Euclídea) entre las distintas categorías permite analizar la relación entre ellas.

La tabla 2 describe cuatro dimensiones estimadas por el modelo de reducción por escalonamiento óptimo. El autovalor (eigenvalue) indica la proporción de información en el modelo explicado por cada dimensión (Chatfield \& Collins, 1992). Se observa que, en conjunto, las dos primeras dimensiones explican el $78 \%$ de la varianza de la inercia ${ }^{8}$. Ambas dimensiones resultan importantes, ya que explican, relativamente, con igual peso la varianza $(40,99 \%, 37,1 \%)$ confirmando que en estas dimensiones se puede encontrar mayor dependencia entre las variables. Así lo confirma el valor del coeficiente de alfa de Cronbach $(>0,92)$, el cual mide el grado de confiabilidad de la escala de medidas de discriminación y es la media ponderada de las correlaciones entre las variables (Chatfield \& Collins, 1992). Esto indica que las variables observables y latentes que conforman ambas dimensiones están altamente correlacionadas y son interdependientes, lo cual garantiza la pertinencia del análisis de correspondencias. Por consiguiente, estas dimensiones se pueden seleccionar para efectuar el análisis de conglomerados posterior.

Tabla 2. Contribuciones relativas de las dimensiones a la inercia total

\begin{tabular}{crrrr}
\multicolumn{5}{c}{ Model Summary } \\
\hline \multirow{2}{*}{ Dimension } & \multirow{2}{*}{ Cronbach's Apha } & \multicolumn{4}{c}{ Variance Accounted For } \\
\cline { 3 - 6 } & & Total (Eigenvalue) & Inertia & \% of Variance \\
\hline 1 & 0,931 & 9,017 & 0,41 & 40,986 \\
2 & 0,919 & 8,17 & 0,371 & 37,134 \\
3 & 0,905 & 7,359 & 0,334 & 33,449 \\
4 & 0,88 & 6,255 & 0,284 & 28,432 \\
\hline \multicolumn{7}{l}{ Total } & 30,8 & 1,4 & \\
\hline \multicolumn{4}{l}{ Mean } & 7,7 \\
\hline \multicolumn{4}{l}{ a. Mean Cronbach's Alpha is based on the mean Eigenvalue. } \\
\hline
\end{tabular}

Fuente: Arenas, 2013

La figura 1 resume las medidas de discriminación (puntajes) para todas las variables observables en cada una de las dos dimensiones. Estos valores permiten observar visualmente cuánto discrimina cada variable en cada dimensión y cuál es la

8 La inercia es una medida de dispersión de los perfiles fila en el espacio multidimensional (Chatfield \& Collins, 1992) 
importancia relativa de cada variable (figura 1, panel izquierdo) y de las unidades de observación (figura 1, panel derecho) en cada una de las dimensiones. Mientras más lejos del origen mayor es el puntaje y más explicativa es la variable. La cercanía con una u otra dimensión reflejan su relación con esta. Igualmente, la proximidad de un trapiche al eje de la dimensión define sus características.

Como se desprende del panel izquierdo de la figura 1, se comprueba que la primera dimensión está integrada por 10 variables con valores de discriminación o puntajes altos superiores a 0,85 . Estas variables y su puntaje respectivo (en paréntesis) son: mano de obra familiar no remunerada $(0,949)$; fuerza animal no remunerada y fuerza animal remunerada $(0,935)$; semanas de molienda anual del trapiche $(0,934)$; toneladas de panela producida al año $(0,928)$; costo de los activos fijos del trapiche $(0,921)$ y mano de obra total por año $(0,915)$. Variables como: las semanas de molienda con caña de fincas vecinas $(0,852)$, las toneladas de caña procedentes de las fincas vecinas $(0.828)$ y la mano de obra ocasional pagada en efectivo $(0,874)$ resultaron ser también importantes en la definición de la dimensión uno.

Figura 1. Medidas de discriminación de las variables en el plano bidimensional
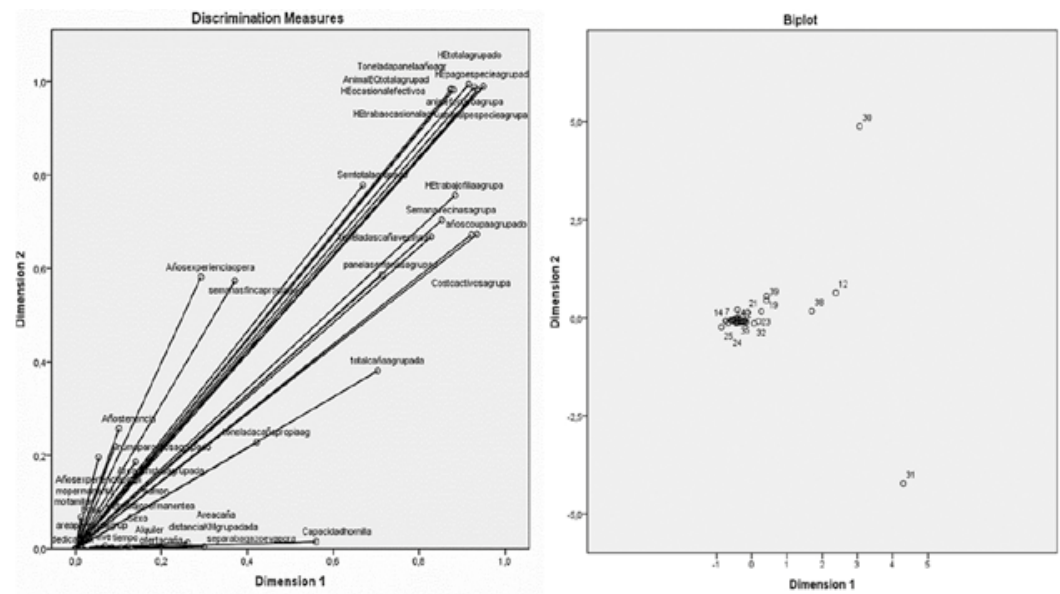

Fuente: Arenas, 2013

Igualmente se corrobora que, la segunda dimensión está conformada fundamentalmente por 7 variables con puntajes muy altos, arriba de 0,98, así: mano de obra total por año $(0,994)$; mano de obra familiar no remunerada $(0,989)$; toneladas de panela producida al año $(0,988)$; mano de obra ocasional pagada en efectivo $(0,983)$; fuerza animal total $(0,982)$ y fuerza animal remunerada y no remunerada $(0,981)$. Se puede ver que estas variables también determinan la dimensión uno pero con puntajes ligeramente menores. Este resultado sugiere que, este grupo de variables, caracterizan en general la estructura productiva y tecnológica de los 
trapiches paneleros de Ocamonte y reflejan que la producción de panela se basa en una tecnología intensiva en el uso de mano de obra y fuerza animal.

Al comparar las dos dimensiones se concluye que los trapiches en la dimensión uno, son altamente dependientes, además de la provisión de cañas de fincas vecinas en volúmenes y con frecuencias de suministros mayores que para las fincas en la dimensión dos. Es decir, estos trapiches tienen una mayor capacidad de molienda, pero no producen en sus propios campos caña suficiente, a fin de darle mayor ocupación a la capacidad instalada de los mismos. Igualmente, estos resultados sugieren que, los trapiches en la dimensión uno, operan más mecanizados con activos fijos de mayor valor relativo y la mano de obra familiar es más importante o crítica para su operación que en los trapiches de la dimensión dos.

Estas dos dimensiones, al integrarse en el análisis, permiten puntualizar algunas de las particularidades del sistema de producción de panela de los trapiches de Ocamonte. La dimensión uno admite un grupo de trapiches operados por productores más organizados y tecnificados para producir panela, que buscan utilizar intensivamente los recursos disponibles (propios, en compañía o por alquiler) de mano de obra, fuerza animal, tierra para caña y capacidad instalada. Se evidencia que, estos trapiches del municipio disponen de una capacidad instalada no utilizada en su totalidad, por cañas producidas en la propia finca.

La dimensión dos permite observar otro grupo de trapiches en los cuales el sistema de producción se caracteriza por: un nivel menor de productividad, mecanización de las operaciones, organización y especialización (artesanal); depende principalmente del trabajo físico de hombres y animales; hace un bajo uso de la capacidad instalada dependiendo la operación de las cañas propias de la finca. Algunos de estos trapiches son operados en alquiler por agricultores productores de caña. Al observarse el panel de la derecha de la figura 1, se devela que la nube de los trapiches de Ocamonte se aproxima más o corresponde principalmente a la dimensión dos. Por tanto, esta dimensión resulta ser la más idónea para definir y caracterizar los sistemas de producción típicos de panela en Ocamonte.

\section{Análisis de conglomerados}

El análisis de correspondencias de la sección anterior permite disponer de información a posteriori sobre cuáles, cómo y porqué las variables de clasificación están más correlacionadas. El análisis de conglomerados, a su vez, facilita el proceso de clasificar los distintos trapiches en grupos homogéneos o tipos, respecto a las variables identificadas con mayor poder clasificatorio, pero lo más distinto posible entre ellos con respecto a estas características. Al efecto los grupos tipo se conformaron mediante un análisis de conglomerados jerárquicos. Este análisis derivó inicialmente en cuatro grupos tipo como se aprecia en el dendograma del panel a) de la figura 2 . 
Figura 2. Distribución de los cuatro grupos de trapiches tipo según dendograma de conglomeración (panel a) y el mapa territorial (panel b)
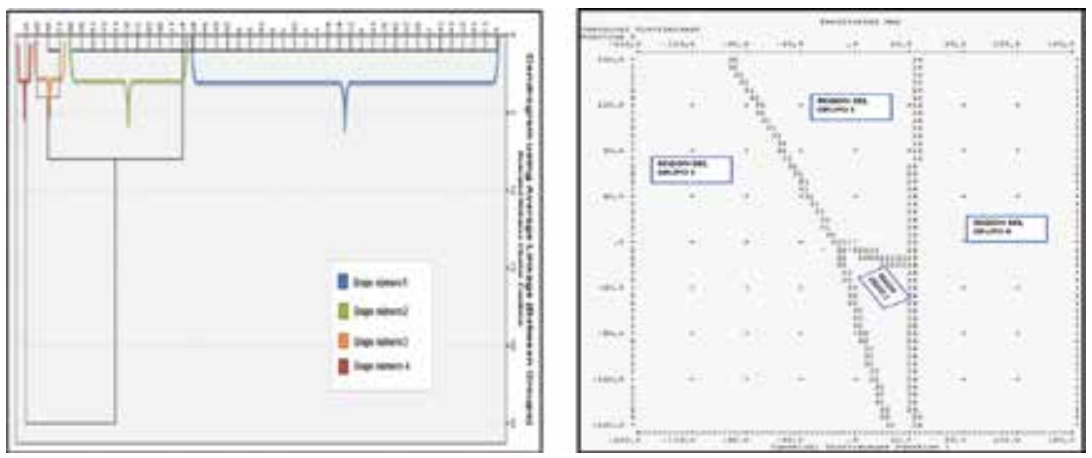

Fuente: Arenas, 2013.

\section{Análisis discriminante}

Los grupos tipo derivados del análisis de conglomerados se corroboraron estadísticamente como bien conformados, mediante un análisis discriminante adelantado en dos pasos. En el primero se ubicaron las observaciones de acuerdo con los conglomerados, corroborando la clasificación hecha en el análisis de conglomerados anterior. En el segundo, y dadas las funciones discriminantes obtenidas, se posibilitó la ubicación de otras unidades no incluidas en este estudio. Este análisis utilizó como variables de clasificación el conglomerado de pertenencia ${ }^{9}$ y como variables independientes las dimensiones 1 y 2 resultantes del análisis de correspondencias.

La tabla 3 ilustra el valor del estadístico Lambda de Wilks, el cual es estadísticamente significativo para ambas variables sintéticas o dimensiones (su valor es menor al nivel de $\mathrm{P}<0,05)$. A su vez, la prueba de $\mathrm{F}$ de entrada es superior al valor crítico de 3,82 , lo cual indica que ambas dimensiones pueden ser seleccionadas como dependientes para describir y discriminar o conformar los grupos. Este resultado permite concluir que, la información aportada por ambas dimensiones, es importante para clasificar las observaciones y sus respectivas funciones tienen poder discriminante.

La distribución de regiones que corresponde a cada tipo de trapiches se ilustra en el panel b) de la figura 2. La distribución de los trapiches dentro de cada grupo fue la siguiente: Grupo 1. Conformado por 28 trapiches, identificados por los números: 1, $2,3,4,5,6,7,8,9,10,13,14,15,16,18,22,24,25,26,27,28,29,34,35,37,40,42$ y 43; Grupo 2. Integrado por 11 trapiches: 11, 17, 19, 20, 21, 23, 32, 33, 36, 39 у 41; Grupo 3. Configurado por 3 trapiches: 12, 30 y 38; Grupo 4: constituido por un solo

9 Variable que se crea luego del análisis de clasificación jerárquica. Sus valores indican el conglomerado final al que pertenece cada caso o individuo (Chatfield \& Collins, 1992). 
trapiche: 31. Luego de validada la selección de los tipos, se evaluó la tipificación y determinaron las variables discriminantes que permiten clasificar nuevas unidades de trapiches no incluidas en este estudio.

Tabla 3. Variables sintéticas o dimensiones introducidas en el modelo

\begin{tabular}{|c|c|c|c|c|c|c|c|c|c|}
\hline \multicolumn{10}{|c|}{ Variables Entered/Removed ${ }^{a, b, c, d}$} \\
\hline \multirow{3}{*}{ Step } & \multirow{3}{*}{ Entered } & \multicolumn{8}{|c|}{ Wilks' Lambda } \\
\hline & & \multirow{2}{*}{ Statistic } & \multirow{2}{*}{ df1 } & \multirow{2}{*}{ df2 } & \multirow{2}{*}{ df3 } & \multicolumn{4}{|c|}{ Exact $\mathbf{F}$} \\
\hline & & & & & & Statistic & df1 & df 2 & Sig. \\
\hline 1 & $\begin{array}{c}\text { Object scores } \\
\text { dimension } 2\end{array}$ & 0,015 & 1 & 3 & 39 & 859,148 & 3 & 39 & 0 \\
\hline 2 & $\begin{array}{l}\text { Object scores } \\
\text { dimension } 1\end{array}$ & 0,001 & 2 & 3 & 39 & 371,312 & 6 & 76 & 0 \\
\hline \multicolumn{10}{|c|}{ At each step, the variable that minimizes the overall Wilks' Lambda is entered. } \\
\hline \multicolumn{10}{|c|}{ a. Maximum number of steps is 4 . } \\
\hline \multicolumn{10}{|c|}{ b. Minimum partial $\mathrm{F}$ to enter is 3.84 . } \\
\hline \multicolumn{10}{|c|}{ c. Maximum partial $\mathrm{F}$ to rem ove is 2.71 . } \\
\hline d. F lev & tolerance, or $\mathrm{VI}$ & $\mathrm{N}$ insufficie & for $\mathrm{fu}$ & er con & ation. & & & & \\
\hline
\end{tabular}

Fuente: Arenas, 2013

Del análisis se derivó que, la función con mayor poder discriminante, era la función discriminante uno (D1). Como lo corrobora el estadístico Lambda de Wilks en la tabla 4, esta función explicaba el $97,7 \%$ de la variabilidad del modelo. En síntesis, la discriminación es aceptable estadísticamente para las dos funciones (D1 y D2).

Tabla 4. Resumen de funciones canónicas discriminantes

\begin{tabular}{ccccc}
\multicolumn{5}{c}{ Eigenvalues } \\
\hline Function & Eigenvalue & \% of Variance & $\begin{array}{c}\text { Cumulative } \\
\text { \% }\end{array}$ & $\begin{array}{c}\text { Canonical } \\
\text { Correlation }\end{array}$ \\
\hline 1 & $175,510^{\mathrm{a}}$ & 97,7 & 97,7 & 0,997 \\
2 & $4,206^{\mathrm{a}}$ & 2,3 & 100 & 0,899 \\
\hline
\end{tabular}

a. First 2 canonical discriminant functions were used in the analysis.

Wilks' Lambda

\begin{tabular}{ccccc}
\hline $\begin{array}{c}\text { Test of } \\
\text { Function(s) }\end{array}$ & $\begin{array}{c}\text { Wilks' } \\
\text { Lambda }\end{array}$ & Chi-square & df & Sig. \\
\hline 1 through 2 & 0,001 & 266,106 & 6 & 0 \\
2 & 0,192 & 64,344 & 2 & 0 \\
\hline
\end{tabular}

Fuente: Arenas, 2013. 
Una de las aplicaciones del análisis discriminante es que las funciones respectivas pueden utilizarse para clasificar nuevos trapiches en el futuro o trapiches actuales no incluidos en la muestra. Los valores estandarizados de estas funciones para cada dimensión fueron las siguientes:

\section{D1: -1,265 dimensión1 + 1,581 dimensión2 \\ D2: 0,951 dimensión1 + 0,062 dimensión2}

Con el fin de establecer cuáles son los tipos de trapiches que se puedan encontrar en el futuro en Ocamonte, se calcularon las probabilidades a priori de conformación de cada tipo de trapiche. Como resultado se obtuvo, que la probabilidad de encontrar trapiches del Tipo I es la más alta, seguida del Tipo II, y en menor medida de los tipos III y IV. Las probabilidades a priori de conformación de los grupos se ilustran en la tabla 5.

Tabla 5. Probabilidades a priori de los tipos de trapiches en Ocamonte

\begin{tabular}{lrrrr}
\multicolumn{4}{c}{ Prior probabilities for groups } \\
\hline \multirow{2}{*}{$\begin{array}{c}\text { Average Linkage } \\
\text { (Between Groups) }\end{array}$} & Prior & \multicolumn{2}{c}{ Cases Used in Analysis } \\
\cline { 3 - 5 } & 1 & 0,907 & Unweighted & Weighted \\
\hline & 2 & 0,047 & 2 & 39 \\
& 3 & 0,023 & 1 & 2 \\
& 4 & 0,023 & 1 & 1 \\
Total & 1 & 43 & 1 \\
\hline
\end{tabular}

Fuente: Arenas, 2013.

Con ayuda de las funciones discriminantes (D1 y D2) y de las probabilidades a priori de conformación de cada Tipo, se pueden clasificar otros trapiches que existan en la zona. Como se observa en la tabla 6 , una nueva observación se clasifica en el Tipo cuyo centroide esté más cerca de la puntuación discriminante ${ }^{10}$ de la observación. Es decir, las funciones discriminantes permiten que cada nuevo trapiche se clasifique en aquel Tipo al cual tenga mayor probabilidad a priori de pertenecer. En síntesis los trapiches (nuevos o futuros) que tengan puntuaciones discriminantes similares, se situarán próximos a las puntuaciones de los Tipos de trapiches ya definidos, según sean los valores de las funciones discriminantes de cada uno.

10 Las puntuaciones discriminantes, son los valores de las funciones discriminantes para cada variable sintética o dimensión (Chatfield \& Collins, 1992). 
Tabla 6. Funciones en los centroides de los grupos

\begin{tabular}{lcr}
\hline \multicolumn{3}{c}{ Canonical discriminant function coefficients } \\
\cline { 2 - 4 } & \multicolumn{3}{c}{ Function } \\
\cline { 2 - 4 } & 1 & 2,118 \\
\hline Object scores & $-2,817$ & \\
dimension 1 Object & & 0,484 \\
scores dimension 2 & 12,335 & \\
(Constant) & & 0
\end{tabular}

Unstandardized coefficients

Fuente: Arenas, 2013.

\section{Descripción de los tipos de trapiches}

La anterior clasificación estadística se corroboró en el campo mediante visitas a una muestra de trapiches agrupados según la tipología, concluyéndose que los cuatro tipos de trapiches conformados eran una adecuada simplificación de la heterogeneidad de la producción panelera en Ocamonte. Igualmente se constató en la realidad que, las variables de tipificación seleccionadas eran las principales características con poder de discriminación entre los trapiches observados. La tabla 7 resume el valor promedio de las principales variables que caracterizaron los diferentes tipos de trapiches de la muestra.

Tabla 7. Estadísticos descriptivos de las variables de tipificación de los trapiches según las dimensiones 1 y 2

\begin{tabular}{lccccc}
\hline \multirow{2}{*}{ Variable } & \multicolumn{3}{c}{ Tipología de trapiches } \\
\cline { 2 - 6 } & Unidad & I & II & III & IV \\
\hline Proporción de trapiches de la muestra & $\%$ & 65 & 22 & 7 & 2 \\
Rango de experiencia del productor & años & $25-40$ & $5-15$ & $25-40$ & $25-40$ \\
Tenencia del trapiche & años & 32,5 & 10 & 32,5 & 40 \\
Semanas molienda total & \#/año & 6 & 16 & 26 & 44 \\
Semanas molienda en alquiler total & \#/año & 2 & 3 & 12 & 33 \\
Capacidad hornilla total & Kg/hora & 75 & 150 & 200 & 200 \\
Utilización capacidad instalada molienda & $\% / a n ̃ o ~$ & 13,5 & 36,6 & 66,0 & 100 \\
Cañas propias molidas total & $\% / a n ̃ o$ & 7 & 100 & 54 & 25 \\
Producción promedia panela total & Ton/año & 70 & 264 & 584 & 1.000 \\
Mano de obra ocupada total & EH/año & 5,26 & 17,88 & 40,23 & 73,6
\end{tabular}




\begin{tabular}{lccccc} 
Mano de obra ocasional ocupada total & EH/año & 4,9 & 9,42 & 38,5 & 71,7 \\
Mano de obra familiar ocupada total & EH/año & 0,36 & 8,46 & 1,65 & 1,9 \\
Mano de obra pagada en especie total & $\%$ & 44 & 40 & 39 & 37 \\
Mano de obra pagada en efectivo total & $\%$ & 56 & 60 & 61 & 63 \\
Fuerza animal ocupada total & EA/año & 1,86 & 8,28 & 24,35 & 53,5 \\
Fuerza animal en alquiler ocupada total & \%/año & 90 & 77 & 100 & 100 \\
Fuerza animal pagada en efectivo total & \%/año & 13 & 13 & 13 & 13 \\
Fuerza animal pagada en especie total & \%/año & 87 & 87 & 87 & 87 \\
Valor promedio activos & \$ Millón & $>300$ & $300-<600$ & $600-<900$ & $>900$ \\
\hline
\end{tabular}

Fuente: Arenas, 2013.

\section{Tipo I: Sistema de trapiche marginal}

Este tipo comprende el $65 \%$ de los trapiches estudiados. El grupo se caracteriza por tener una dotación de recursos y servicios deficiente y una estructura y organización productiva y tecnológica con severas carencias, representadas esencialmente por instalaciones rústicas y uso de prácticas tradicionales del proceso, el molino y la hornilla en su totalidad. La mano de obra familiar disponible es muy baja. La frecuencia de molienda no alcanza a ser mensual, debido a la restringida oferta de caña, tanto de la misma finca como de las fincas vecinas. Por tanto, la utilización de la capacidad instalada es en promedio inferior al 13,5\%. Estas unidades cuentan con una superficie sembrada en caña pequeña menor a 5 ha en promedio y la operación depende de la mano de obra familiar y mulas contratadas.

\section{Tipo II: Sistema de trapiche tradicional}

Este grupo constituye el $26 \%$ de los trapiches en la muestra y corresponde a unidades con una extensión de caña propia relativamente alta (promedio de 48 hectáreas). Por tanto, procesan principalmente cañas provenientes de sus sembradíos y muelen al menos una semana al mes para una utilización de la capacidad instalada del 36,6\%. La mayoría de la mano de obra familiar vive y trabaja en la finca. Sin embargo, son trapiches con una dotación física y tecnológica tradicionales. Se diferencia de los demás grupos porque los productores tienen menos años de tenencia del predio y cuentan con disponibilidad de mulas propias en la finca para el transporte de la caña. No obstante, la operación manual utiliza mano de obra contratada.

\section{Tipo III: Sistema de trapiche mejorado}

Este sistema lo compone el 7\% de los trapiches de la muestra. Se caracterizan por contar con una dotación de recursos físicos y tecnológicos mejorados respecto al tipo II. Los productores son propietarios de tierra, experimentados y operan parcialmente la unidad productiva, con pocos propietarios viviendo en el predio. No 
poseen trapiche propio y deben moler la caña en trapiches rentados o en el sistema de maquila. La oferta de caña propia es la mitad de la molida. El resto proviene de cañas vecinas. En estas unidades el trabajo animal es realizado con mulas en alquiler o "fletadas" en su totalidad.

\section{Tipo IV: Sistema de trapiche avanzado}

Este sistema se compone únicamente por una unidad productiva. Se considera como un trapiche avanzado en función de la moderna dotación de recursos físicos, tecnología de producción y procesos, administración, organización y desempeño productivo. El productor y la familia viven en la unidad. La utilización de la capacidad instalada es del 100\% y su operación es relativamente continua a lo largo del año con solo el $25 \%$ de la caña molida procedente de tierras propias y el resto de fincas vecinas. Esta unidad es intensiva en el uso de mano obra contratada

\section{Conclusiones y recomendaciones}

El presente estudio es un trabajo piloto de aplicación de una metodología robusta para conformar grupos homogéneos de trapiches en Ocamonte, Santander. Los resultados de la tipología y su validación estadística y empírica en el campo demuestran que, existe una alta heterogeneidad en las condiciones de producción de la panela. También sustentan que, el uso de las técnicas estadísticas del análisis multivariado es apropiado para tipificar trapiches paneleros, reduciendo la variabilidad a tipos de trapiches con características técnicas y socioeconómicas de los productores similares.

La actividad panelera en Ocamonte se caracteriza por el uso intensivo del factor capital de inversión y de operación, y de los factores mano de obra y fuerza animal. El capital de inversión es aportado por el propietario. El trabajo familiar y los gastos de operación los aporta el productor y cónyuge. La contratación de mano de obra permanente es baja y mantiene relación directa con la ausencia del propietario en la unidad. La mano de obra ocasional es contratada por molienda y su participación en el costo de operación es el más elevado. La fuerza animal está representada por el trabajo de equinos para el transporte de caña verde desde los lotes al trapiche. La mayoría de los equinos se alquilan y pagan mediante contratos informales entre propietarios de los animales y de las cañas a moler o el dueño del trapiche o de la molienda.

Desde el punto de vista metodológico se encontraron dos dimensiones o ejes principales para tipificar los trapiches. La primera dimensión describe a los trapiches, como unidades productivas, que se organizan para producir panela mediante el manejo de los recursos de capital en instalaciones y equipos y de operación en insumos y mano de obra; producción y compra de caña, contratación de mano de obra y fuerza animal (propia, en asocio o por alquiler) con el fin de lograr ingresos 
para cubrir las necesidades básicas de auto subsistencia. La segunda, describe la respuesta de los trapiches en términos de los niveles de organización, propiedad del trapiche, dotación de caña panelera, recursos y servicios de apoyo, tecnología y producción con destino al mercado.

La producción de caña y panela en Ocamonte claramente queda delimitada a tres sistemas diferenciados de producción. En primer término están los aparceros o trabajadores sin tierra ni trapiche, quienes cultivan caña en compañía con los propietarios de los trapiches o la tierra, y comparten los gastos y el producido de panela en forma proporcional (trapiches Tipo I). En segundo lugar están aquellos que son propietarios de la tierra y cultivan caña, pero no son dueños de trapiche y deben tomar en alquiler los trapiches (trapiches Tipo III). Por último, están los dueños de trapiche quienes cultivan sus propias plantaciones de caña y a la vez realizan la molienda de cañas propias, usando una tecnología tradicional (trapiches Tipo II). Finalmente, están los trapiches operados por el propietario, quienes muelen cañas propias y de vecinos empleando una tecnología avanzada (trapiches Tipo IV).

La tipificación evidencia la existencia de amplias brechas de eficiencia productiva entre los trapiches. El análisis arroja que los tipos de trapiche se diferencian debido a: variaciones en su localización (altura sobre el nivel del mar: 800 a 2000 m), el nivel de inversiones, operación, mecanización, organización y especialización del proceso (artesanal versus empresarial); y variaciones en la productividad y disponibilidad de caña y de trabajo físico (hombres y animales). Todo esto se traduce en amplias variaciones en el nivel de eficiencia productiva y de escala de operación, lo cual sugiere la presencia de amplias brechas de productividad entre los tipos II y IV y el resto de trapiches. Cerrar estas brechas mediante adecuada transferencia de tecnología, debe contribuir a aumentar la productividad y reducir el costo unitario de la panela en el corto plazo.

El estudio resulta oportuno, en el contexto del nuevo programa de desarrollo rural con enfoque territorial en Colombia (Berdegué, Castro, González, \& Perry, 2014). Para su adecuado diseño, implementación y evaluación se requiere disponer de información básica que oriente el diseño de líneas de política y la formulación de instrumentos para la inclusión productiva y social de los habitantes del sector rural. En este sentido, la metodología validada para generar información robusta sobre la fase agroindustrial de producción de panela, es esencial en la búsqueda de estrategias para aumentar los bajos niveles de productividad, calidad, competitividad y bienestar actuales de la mayoría de productores, familias y comunidades paneleras.

Como consecuencia de lo anterior, se recomienda: adelantar la definición de las zonas óptimas para la producción de caña y panela en el municipio, ya que algunas zonas son marginales para la producción de caña panelera; impulsar nuevas formas de organización de los productores con enfoques socio organizativos y de mercado o agroempresariales; promover la consecuente diversificación de la producción de caña en las zonas no óptimas y la intensificación del cultivo de caña en las zonas 
óptimas; investigar la factibilidad técnica y financiera de la reconversión de buena parte de los trapiches mediante centros comunales de acopio, beneficio y distribución. Finalmente, reorientar la investigación y transferencia de tecnología (asistencia técnica y extensión agrícola), aumentar la productividad y cerrar las brechas de productividad en trapiches y campos de caña panelera.

\section{Referencias}

Acosta, S. E., \& Pérez , J. (2011). The Colombian Agricultural Statistics System under a new conceptualization. Wye City Group Statistics on Rurall Development and Agricultural Hose Income IV meeting (p. 27). Río de Janeiro, Brazil: Wye City Group.

Aguiar, S. B. (2001). Craterización y tipificación de los sistemas de producción de caña en el municipio de Tamara, Casanare. Corpoica Informe Técnico No. 33, $1-48$.

Arenas, L. X. (2013). Estudio piloto para la tipificación técnica y socioeconómica de trapiches paneleros en el municipio de Ocamonte, Santander. (Tesis de grado Economista) Universidad Santo Tomás. Bucaramanga, Colombia.

Berdegué, J., Castro, F., González, E., \& Perry, S. (2014). Estrategia de implementación del Programa de Desarrollo Rural Integral con Enfoque Territorial. Bogota, Colombia: Centro Interamericano de Desarrollo Rural RIMISP.

Calvo, G., \& Icaza, J. (1988). Técnicas de análisis multivariado y de programación lineal en la evaluación de alternativas tecnológicas mejoradas a nivel de finca: el caso de Esteli, Nicaragua. En G. Escobar, \& J. Berdegué. Clasificación de sistemas de finca para generación y transferencia de tecnología apropiada (pp. 109-119). IDRC Ottawa: Escobar Germán.

Capillon, A. (1986). A classification of farming systems, preliminary to an extension program. In Methodology farming systems research and extension: management and methodology (pp. 219-235). Manhattan: Kansas State University.

Carvajal, G., Pinzon, L. M., \& Restrepo, G. (1992). Tipificación de fincas en la zona ganadera del Magdalena Medio oeste. Manizales: ICA: Regional.

Chatfield, C., \& Collins, A. J. (1992). Introduction to Multivariate Statistical Analysis. London: Chapman \& Hall.

Clausen, E. (1998). Applied Correspondence Analysis. Thousand Oaks, CA: Sage Publications.

Cohan, E., \& Alonso, A. (1977). Aplicación de técnicas estadísticas para la tipificación de empresas agropecuarias. I Seminário de Modernicao da Empresa Rural. Río de Janeiro: IICA. 
DANE. (2009). La estadística estratégica del sector agropecuario en Colombia: un nuevo modelo de oferta. Revista de la Información Básica 3(2), 1-14.

DNP-MTC. (2015). Diagnóstico económico del campo colombiano: informe de la MTC. Bogotá DC: DNP.

Dussan, C. (1996). Análisis estadístico multivariadod de fincas cafeteras exitosas en el departamento de Caldas. (Tesis de grado Especialización Estadística). Manizales, Colombia: Universidad Nacional.

Escobar, G., \& Berdegué, J. (1990). Tipificación de sistemas de producción agrícola. Santiago de Chile: RIMISP.

Fedesarrollo-Iquartil-SAC. (2013). Costos de producción de 12 productos agropecuarios. Informe Final Fedesarrollo.

Hair, J., Anderson, R., Tatham, R., \& Black, W. (1998). Multivariate data analysis. (5th ed.). New York, NY: Prentice Hall.

Isaacs, H., Carrillo,. E., Caicedo, H., Paz, G., Palma, E., \& Ramírez, A. (1998). Clientes de la nueva tecnología: Censo y tipificación de caña de azúcar de la industria azucarera colombiana. Serie Técnica, No. 27, 1-86.

Johnson, A., \& Williamson, C. (1998). Multivariate statistical analysis. New York: Prentice Hall.

Kaminski, M. (1982). Metodología de regionalización agropecuaria por tipificación: Una aplicación al caso de Paraguay. Desarrollo Rural en las Américas 14, 23-45.

Kaminski, M. (1986). Enfoque de sistemas de fincas y tipificación de unidades de producción agropecuaria: referencias, comentarios y posiciones. En G. Escobar. Clasificación de Sietemas de finca para generación y transferncia de tecnología apropiada (p. 124). Panamá: CIID.

Landín, R., \& Berdegué, J. (1990). Tipificación de fincas lecheras en Ecuador. En G. Escobar, \& J. Berdegúe. Tipificación de sistemas de producción agrícola (pp. 86-89). Santiago de Chile: RIMISP.

Madry, W., Mena, Y., Rosbowska, B., Gozdowski, D., Hrynieski, R., \& Castel, R. (2013). An overview of farming system typology methodologies and its use in the study of pasture-based farming systems. Spanish Journal of Agricultural Research, 11(2), 316-326.

Mantilla, J., Arguello, A. L., \& Méndez, H. (2000). Caracterización y tipificación de productores de cacao en el departamento de Santander. Bucaramanga: Corpoica.

Manyong, A., Degand, L., D’ Haese, P., \& Dutilleul, P. (1988). Research on a typology of traditional farming in Burundi. Agricultural Systems, 103-117. 
PNUD. (2011). Informe Nacional de Desarrollo Humano 2011: Colombia Rural Razones para la Esperanza. Bogotá: INDH PNUD.

Pretzer, D., \& Finley, R. (1974). Farm type classification systems: another look at an old problem. American Journal of Agricultural Economics, 56, 145-149.

Ríos, G. (1999). Tipificación y caracterización del cultivo del plátano en el departamento de Caldas. (Tesis de grado). Manizales, Colombia: Universidad de Caldas.

Ríos, G., Botero, M. J., Franco, G., Romero, M., Pérez, J. C., Morales, J., Echeverry, D. (2002). Caracterización y tipificación de los sistemas de producción de mora y lulo en el departamento de Caldas. Manizales, Colombia: Corpoica.

Smith, R., Moreira, V., \& Latrille, L. (2002). Caracterización de sistemas productivos lecheros en la X Región de Chile mediante análisis multivariable. Agricultura Técnica, 62(3), 1-21.

Suárez, R., \& Escobar, G. (1990). Tipificación de fincas en la comarca de Fusagasugá, Colombia, según sus tendencias de cambio técnico. En Tipificación de sistemas de producción agrícola (pp. 181-200). Santiago de Chile: RIMISP.

Winkler, A. (1977). Relación entre estructura productiva y sociocultural y uso de la tecnología disponible: un análisis basado en tipificaciones de empresas agropecuarias de la IX región de Chile. Castelar, Argentina: Escuela para Graduados en Ciencias Agropecuarias.

World Bank. (2009). World Bank Report 2008: Agriculture for Development. Whashington D.C.: World Bank. 